\title{
The Trojan Horse Method in Nuclear Astrophysics
}

\author{
Aurora Tumino ${ }^{1,2, \star}$, Claudio Spitaleri ${ }^{2}$, Silvio Cherubini ${ }^{2,3}$, Giuseppe D'Agata ${ }^{2}$, Giovanni Luca \\ Guardo $^{2}$, Marisa Gulino ${ }^{1,2}$, Iolanda Indelicato², Marco La Cognata ${ }^{2}$, Livio Lamia², Rosario \\ Gianluca Pizzone ${ }^{2}$, Giuseppe Gabriele Rapisarda ${ }^{2}$, Stefano Romano ${ }^{2,3}$, Maria Letizia Sergi², \\ and Roberta Spartá 2 \\ ${ }^{1}$ Facoltá di Ingegneria e Architettura, Universitá degli Studi di Enna "Kore", Enna, Italy \\ ${ }^{2}$ INFN-Laboratori Nazionali del Sud, Catania, Italy \\ ${ }^{3}$ Dipartimento di Fisica e Astronomia, Universitá degli Studi di Catania, Catania, Italy
}

\begin{abstract}
The Trojan Horse Method (THM) represents the indirect way to measure reactions between charged particles at astrophysical energies. This is done by measuring the quasi free cross section of a suitable three body process. The basic features of the THM will be presented together with some applications to demonstrate its practical use.
\end{abstract}

\section{Introduction}

The Trojan Horse Method (THM) has been introduced in nuclear astrophysics as an indirect approach to determine low-energy cross sections overcoming the main issues of direct experiments, such as the Coulomb repulsion and the electron screening $[1,2]$. The first one is responsible for the exponential decrease of the cross section at the relevant temperatures, while the electron screening, due to the electrons surrounding the interacting ions, leads to an increased cross section compared to the one for bare nuclei that is necessary to assess the reaction rate in astrophysical plasma.

The THM ([3-6] and references therein) makes use of a suitable $A+a \rightarrow b+B+s$ two-to-three body process to measure the astrophysical $A+x \rightarrow b+B$ two-body reaction of interest by means of a relation between the two based on nuclear reaction theories. The choice of the three-body process is done in such a way that target $a$ (or equivalently the projectile) has a wave function with a large amplitude for a $x-s$ cluster configuration, $x$ being the target (or equivalently the projectile) of the two-body reaction. The selected part of the three-body phase space conveys with the quasi-free (QF) kinematics: the other cluster $s$ remains spectator to the process, and $A+x \rightarrow b+B$ can be regarded as a half-off-energyshell (HOES) two-body reaction, usually referred to as a QF reaction. The QF reaction mechanism is sketched in Fig.1 using a pole diagram with upper and lower vertexes describing the break-up of $a$ and the $A+x \rightarrow b+B$ two-body reaction, respectively.

Since the three-body process occurs at an energy above the Coulomb barrier, the main feature is the real suppression of both Coulomb barrier and screening effects in the HOES two-body cross section. Nevertheless, the quasi-free $A+x$ process can occur even at very low sub-Coulomb energies because the $A+a$ relative motion is compensated for by the $x-s$ binding energy. This is indeed a different approach to the THM [3] compared to the original idea of Baur [7], where the initial velocity

^e-mail: tumino@lns.infn.it 


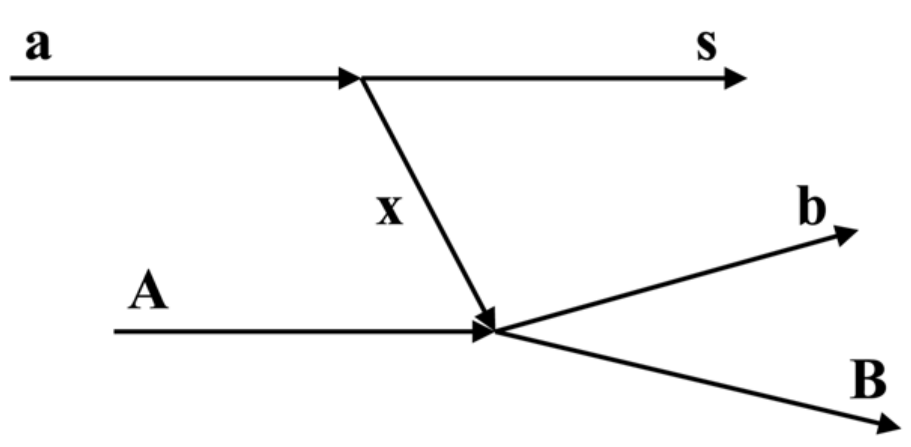

Figure 1. Pole diagram describing the QF mechanism in the $A+a \rightarrow b+B+s$ reaction.

of the projectile $A$ is compensated for by the $x-s$ intercluster motion. In that framework a quite large momentum of the order of $200 \mathrm{MeV} / \mathrm{c}$ or more is needed. But the relative yield of the experimental momentum distribution at such momenta can be very small, in particular for a $1=0$ inter-cluster motion (for example p-n motion inside ${ }^{2} \mathrm{H}$ or $\alpha$-d motion inside ${ }^{6} \mathrm{Li}$ ). This would complicate the separation from other competitive reaction mechanisms. Moreover, the theoretical description of the tails of the momentum distribution is a hard task, their shape being very sensitive to it. In our approach to the THM, the intercluster motion is only needed to determine the accessible astrophysical energy region. It corresponds to a cutoff in momentum distribution of $s$ of few tens of $\mathrm{MeV} / \mathrm{c}$. In this framework, the so called "quasi-free two-body energy" is given by:

$$
E_{Q F}=\frac{m_{x}}{m_{x}+m_{A}} E_{A}-B_{x-s}
$$

where $E_{A}$ represents the beam energy, $m_{x}$ and $m_{A}$ are the masses of $x$ and $A$ particles respectively, and $B_{x-s}$ is the binding energy for the $x-s$ system.

In the Impulse Approximation, based essentially on the assumption that the interaction of the spectator with particles $b$ and $B$ is neglected, the three body-cross cross section can be factorized as:

$$
\frac{d^{3} \sigma}{d E_{b} d \Omega_{b} d \Omega_{B}} \propto\left[K F\left|\varphi_{a}\left(\mathbf{p}_{s x}\right)\right|^{2}\right]\left(\frac{d \sigma}{d \Omega_{c . m .}}\right)^{\mathrm{HOES}}
$$

where KF is a kinematical factor containing the final state phase-space factor. It is a function of the masses, momenta and angles of the outgoing particles [25]; $\varphi_{a}\left(\mathbf{p}_{s x}\right)$ is proportional to the Fourier transform of the radial wave function $\chi(\mathbf{r})$ for the $x-s$ inter-cluster relative motion; $\left(d \sigma / d \Omega_{c . m .}\right)^{\mathrm{HOES}}$ is the half-off-energy-shell (HOES) differential cross section for the binary reaction at the center of mass energy $\mathrm{E}_{c . m}$. given in post-collision prescription by

$$
E_{c . m .}=E_{b B}-Q_{2 b}
$$

Here, $Q_{2 b}$ is the $Q$-value of the binary reaction and $\mathrm{E}_{b B}$ is the relative energy of the outgoing particles $c$ and $C$, which spans the accessible astrophysical region defined above.

The factorization of Eq. 2 is strictly valid in Plane Wave Impulse Approximation, which changes only the absolute magnitude but not the energy dependence of the two-body cross section. 


\section{How to apply the THM}

In THM experiments, any two of the three particles in the exit channel are usually detected and identified by means of telescopes (silicon detector or ionization chamber as $\Delta \mathrm{E}$ detector and PSD as $E$ detector) placed in a phase space region where quasi free kinematics dominates. After the reconstruction of the reaction channel, a critical point is the selection of the quasi free mechanism from other reaction mechanisms feeding the same particles in the final state, e.g. sequential decay and direct break-up. This is done investigating the shape of the experimental momentum distribution $\Phi\left(p_{s}\right)$ of the spectator by means of the energy sharing method [8], for example. The quasi-free coincidence yield with a cutoff in relative energy of few tens of $\mathrm{keV}$ and in center of mass angle of few degrees at most is divided by the kinematic factor, providing a quantity which is proportional to the product of the spectator momentum distribution with the differential two-body cross section (see Eq.2). The window is chosen in such a way that the differential two-body cross section in this range can be considered almost constant. Thus, the quantity defined above represents essentially the momentum distribution for the spectator that in PWIA can be compared with the Fourier transform of the radial $x-s$ bound state wave function.

Data analysis is limited to the region where the agreement between the two distributions is found. Usually a window of few tens of $\mathrm{MeV} / \mathrm{c}$ is chosen not to move too far from $\mathrm{E}_{Q F}$, according to the prescriptions reported in [15].

Therefore, $\left(d \sigma / d \Omega_{c m}\right)^{\mathrm{HOES}}$ can be extracted from the three-body coincidence yield by simply inverting Eq.2. The Coulomb barrier in $\left(d \sigma / d \Omega_{c m}\right)^{\mathrm{HOES}}$ is suppressed [9] and this is due to the virtuality of particle $x$. This seems to be the only consequence of off-energy-shell effects as suggested by the agreement between HOES and OES cross-sections for the ${ }^{6} \mathrm{Li}(\mathrm{n}, \alpha)^{3} \mathrm{H}$ reaction [10].

Thus, in a final step, to relate the HOES excitation function to the relevant on-energy-shell (OES) one, Coulomb corrections have to be considered. If one looks at the angular distributions no correction is needed because once the energy is fixed, it would mean to introduce simply a scaling factor. Thus, the OES data are directly comparable with HOES ones projected onto the emission angle of $C$ (or $c$ ) in the $C-c$ center of mass system, $\theta_{c . m}$, as given by [14]:

$$
\theta_{c . m .}=\arccos \frac{\left(\mathbf{v}_{\mathrm{A}}-\mathbf{v}_{\mathbf{x}}\right) \cdot\left(\mathbf{v}_{\mathbf{c}}-\mathbf{v}_{\mathrm{C}}\right)}{\left|\mathbf{v}_{\mathrm{A}}-\mathbf{v}_{\mathbf{x}}\right|\left|\mathbf{v}_{\mathbf{c}}-\mathbf{v}_{\mathrm{C}}\right|}
$$

where the vectors $\mathbf{v}_{\mathrm{A}}, \mathbf{v}_{\mathbf{x}}, \mathbf{v}_{\mathbf{c}}, \mathbf{v}_{\mathrm{C}}$ are the velocities of projectile, transferred particle and outgoing nuclei respectively. These quantities are calculated from their corresponding momenta in the lab-system, where the momentum of the transferred particle is equal and opposite to that of $s$ when the quasi-free break-up takes place in the target, otherwise a little different formula has to be used [14]. If HOES data are projected onto the $\mathrm{E}_{C c}$ axis, Coulomb suppression has to be introduced before comparison with OES data $[11,12]$. In a simple approach, this is done by means of the penetrability factor:

$$
P_{l}\left(k_{A x} R\right)=\frac{1}{G_{l}^{2}\left(k_{A x} R\right)+F_{l}^{2}\left(k_{A x} R\right)}
$$

with $F_{l}$ and $G_{l}$ regular and irregular Coulomb wave functions and $R$ the so called cutoff radius, which is usually chosen as the sum of the radii of nuclei $A$ and $x$. This procedure does not allow us to extract the absolute value of the two-body cross section. However, this is not a real problem since the absolute magnitude can be derived from a scaling to the direct data available at higher energies.

If a resonant two-body reaction is involved, a dedicated approach called Modified R-matrix ([16] and references therein) has been developed to extract the resonance parameters from the THM reaction yield. The considerable advantage is that they are the same needed to calculated the OES cross section. 
Table 1. Two-body reactions studied via the THM with measured two-to-three TH reaction and relevant references for each reaction

\begin{tabular}{|c|c|c|}
\hline Direct reaction & TH reaction & ref \\
\hline${ }^{7} \mathrm{Li}(\mathrm{p}, \alpha){ }^{4} \mathrm{He}$ & ${ }^{7} \operatorname{Li}(\mathrm{d}, \alpha \alpha) \mathrm{n}$ & [17-19] \\
\hline${ }^{7} \mathrm{Li}(\mathrm{p}, \alpha){ }^{4} \mathrm{He}$ & ${ }^{7} \mathrm{Li}\left({ }^{3} \mathrm{He}, \alpha \alpha\right){ }^{2} \mathrm{H}$ & [20] \\
\hline${ }^{6} \mathrm{Li}(\mathrm{d}, \alpha){ }^{4} \mathrm{He}$ & ${ }^{6} \mathrm{Li}\left({ }^{6} \mathrm{Li}, \alpha \alpha\right){ }^{4} \mathrm{He}$ & [21] \\
\hline${ }^{6} \mathrm{Li}(\mathrm{p}, \alpha){ }^{3} \mathrm{He}$ & ${ }^{6} \mathrm{Li}\left(\mathrm{d}, \alpha{ }^{3} \mathrm{He}\right) \mathrm{n}$ & [22-24] \\
\hline${ }^{11} \mathrm{~B}(\mathrm{p}, \alpha)^{8} \mathrm{Be}$ & ${ }^{11} \mathrm{~B}\left(\mathrm{~d},{ }^{8} \mathrm{Be} \alpha\right) \mathrm{n}$ & {$[25,26]$} \\
\hline${ }^{10} \mathrm{~B}(\mathrm{p}, \alpha){ }^{7} \mathrm{Be}$ & ${ }^{10} \mathrm{~B}\left(\mathrm{~d},{ }^{7} \mathrm{Be} \alpha\right) \mathrm{n}$ & [27-29] \\
\hline${ }^{9} \mathrm{Be}(\mathrm{p}, \alpha){ }^{6} \mathrm{Li}$ & ${ }^{9} \mathrm{Be}\left(\mathrm{d},{ }^{6} \mathrm{Li} \alpha\right) \mathrm{n}$ & {$[30,31]$} \\
\hline${ }^{2} \mathrm{H}\left({ }^{3} \mathrm{He}, \mathrm{p}\right){ }^{4} \mathrm{He}$ & ${ }^{6} \mathrm{Li}\left({ }^{3} \mathrm{He}, \mathrm{p} \alpha\right){ }^{4} \mathrm{He}$ & {$[32]$} \\
\hline${ }^{2} \mathrm{H}(\mathrm{d}, \mathrm{p}){ }^{3} \mathrm{H}$ & ${ }^{2} \mathrm{H}\left({ }^{6} \mathrm{Li}, \mathrm{t} p\right){ }^{4} \mathrm{He}$ & [33-35] \\
\hline${ }^{15} \mathrm{~N}(\mathrm{p}, \alpha){ }^{12} \mathrm{C}$ & ${ }^{15} \mathrm{~N}\left(\mathrm{p}, \alpha^{12} \mathrm{C}\right) \mathrm{n}$ & {$[36]$} \\
\hline${ }^{18} \mathrm{O}(\mathrm{p}, \alpha){ }^{15} \mathrm{~N}$ & ${ }^{18} \mathrm{O}\left(\mathrm{p}, \alpha^{15} \mathrm{~N}\right) \mathrm{n}$ & {$[37,38]$} \\
\hline${ }^{1} \mathrm{H}(\mathrm{p}, \mathrm{p}){ }^{1} \mathrm{H}$ & ${ }^{2} \mathrm{H}(\mathrm{p}, \mathrm{pp}) \mathrm{n}$ & {$[11,12]$} \\
\hline${ }^{2} \mathrm{H}(\mathrm{d}, \mathrm{p}){ }^{3} \mathrm{H}$ & ${ }^{2} \mathrm{H}\left({ }^{3} \mathrm{He}, \mathrm{t} p\right){ }^{1} \mathrm{H}$ & [39-41] \\
\hline${ }^{2} \mathrm{H}(\mathrm{d}, \mathrm{n}){ }^{3} \mathrm{He}$ & ${ }^{2} \mathrm{H}\left({ }^{3} \mathrm{He},{ }^{3} \mathrm{He} \mathrm{n}\right){ }^{1} \mathrm{H}$ & [39-41] \\
\hline${ }^{19} \mathrm{~F}(\mathrm{p}, \alpha){ }^{16} \mathrm{O}$ & ${ }^{2} \mathrm{H}\left({ }^{19} \mathrm{~F}, \alpha^{16} \mathrm{O}\right) \mathrm{n}$ & {$[42,43]$} \\
\hline${ }^{17} \mathrm{O}(\mathrm{p}, \alpha){ }^{14} \mathrm{~N}$ & ${ }^{2} \mathrm{H}\left({ }^{17} \mathrm{O}, \alpha^{14} \mathrm{~N}\right) \mathrm{n}$ & [44] \\
\hline${ }^{4} \mathrm{He}\left({ }^{12} \mathrm{C},{ }^{12} \mathrm{C}\right){ }^{4} \mathrm{He}$ & ${ }^{6} \mathrm{Li}\left({ }^{12} \mathrm{C}, \alpha^{12} \mathrm{C}\right)^{2} \mathrm{H}$ & {$[45]$} \\
\hline $\mathrm{n}\left({ }^{6} \mathrm{Li}, \mathrm{t}\right)^{4} \mathrm{He}$ & ${ }^{2} \mathrm{H}\left({ }^{6} \mathrm{Li}, \mathrm{t}^{4} \mathrm{He}\right){ }^{1} \mathrm{H}$ & {$[10,46]$} \\
\hline${ }^{13} \mathrm{C}(\alpha, \mathrm{n}){ }^{16} \mathrm{O}$ & ${ }^{6} \mathrm{Li}\left({ }^{13} \mathrm{C}, \mathrm{n}^{16} \mathrm{O}\right){ }^{2} \mathrm{H}$ & [16] \\
\hline${ }^{18} \mathrm{~F}(\mathrm{p}, \alpha){ }^{15} \mathrm{O}$ & ${ }^{2} \mathrm{H}\left({ }^{18} \mathrm{~F}, \alpha^{15} \mathrm{O}\right) \mathrm{n}$ & [47] \\
\hline${ }^{19} \mathrm{~F}(\alpha, \mathrm{p}){ }^{22} \mathrm{Ne}$ & ${ }^{6} \mathrm{Li}\left({ }^{19} \mathrm{~F}, \mathrm{p}^{22} \mathrm{Ne}\right){ }^{2} \mathrm{H}$ & [48] \\
\hline${ }^{7} \mathrm{Be}(\mathrm{n}, \alpha){ }^{4} \mathrm{He}$ & ${ }^{2} \mathrm{H}\left({ }^{7} \mathrm{Be}, \alpha \alpha\right){ }^{1} \mathrm{H}$ & [49] \\
\hline${ }^{12} \mathrm{C}\left({ }^{12} \mathrm{C}, \alpha\right){ }^{20} \mathrm{Ne}$ & ${ }^{12} \mathrm{C}\left({ }^{14} \mathrm{~N}, \alpha^{20} \mathrm{Ne}\right){ }^{2} \mathrm{H}$ & [50] \\
\hline${ }^{12} \mathrm{C}\left({ }^{12} \mathrm{C}, \mathrm{p}\right){ }^{23} \mathrm{Na}$ & ${ }^{12} \mathrm{C}\left({ }^{14} \mathrm{~N}, \mathrm{p}^{23} \mathrm{Na}\right)^{2} \mathrm{H}$ & {$[50]$} \\
\hline
\end{tabular}

\section{Resent results}

The THM has applied already many times to resonant and non resonant charged particle reactions connected with fundamental astrophysical problems, from primordial to stellar nucleosynthesis. A list of reactions studied by means of the THM is given in Table 1 together with the relevant references.

Given the well assessed THM experimental and theorerical methodology to investigate low-energy nuclear reactions, applications in the near future are forseen for neutron induced reactions on radioactive nuclei. Having a radioactive beam, deuteron targets will serve as a virtual source of neutrons. This turns out to be a unique way to investigate such reactions. Moreover, after the very successful 
work to determine the C-burning reaction rate, accepted for publication in Nature [50], the THM will be further applied to other key processes triggering the evolution of massive stars, such as ${ }^{12} \mathrm{C}+{ }^{16} \mathrm{O}$ and ${ }^{16} \mathrm{O}+{ }^{16} \mathrm{O}$.

\section{References}

[1] H.J. Assenbaum et al., Z . Phys. A 327461 (1987)

[2] F. Strieder et al., Naturwissenschaften 88461 (2001)

[3] C. Spitaleri et al., Phys. At. Nucl., 74, 1763, (2011)

[4] A. Tumino et al., Few Body Syst., 54, Issue 5-6 745 (2013)

[5] A. Tumino et al., Few Body Syst., 54, Issue 7-10 869 (2013)

[6] Tribble R. et al., Rep. Prog. Phys. 77, Issue: 10106901 (2014)

[7] G. Baur, Phys. Lett. B 178135 (1986)

[8] J. Kasagi et al., Nucl. Phys. A 239233 (1975)

[9] A.M. Mukhamedzhanov et al., Eur. Phys. J. A 27205 (2006)

[10] A. Tumino et al., Eur. Phys. J. A 25649 (2005)

[11] A. Tumino et al., Phys. Rev. Lett. 98252502 (2007)

[12] A. Tumino et al., Phys. Rev. C 78064001 (2008)

[13] M. La Cognata et al., Astrophysical J. 777, 143 (2013)

[14] M. Jain et al., Nucl. Phys. A 15349 (1970)

[15] Shapiro, I.S. et al., Nucl. Phys. A, 61, 353 (1965)

[16] M. La Cognata et al., Phys. Rev. Lett. 49015106 (2013)

[17] C. Spitaleri et al., Phys. Rev. C 60055802 (1999)

[18] M. Aliotta et al. 2000 Eur. Phys. J. A 9, 435

[19] M. Lattuada et al., Ap.J. 562, 1076 (2001)

[20] A.Tumino et al., Eur. Phys. J. A direct, 1 (2006) DOI: 10.1140/epja/i2006-08-038-1

[21] C. Spitaleri et al., Phys. Rev. C 63005801 (2001)

[22] A. Tumino et al., Phys. Rev. C 67065803 (2003)

[23] A. Tumino et al., Prog. Theor. Phys. Suppl. 154341 (2004)

[24] L. Lamia et al., Astrophysical J. 76865 (2013)

[25] C. Spitaleri et al., Phys. Rev. C 63055806 (2004)

[26] L. Lamia et al., Journ. of Phys. G: Nucl. Part. Phys. 39015106 (2012)

[27] L. Lamia et al., Nucl. Phys. A 787309 (2007)

[28] L. Lamia et al., Il Nuovo Cimento 31 N.4 423 (2008)

[29] C. Spitaleri et al., Phys. Rev. C 95035801 (2017)

[30] Qun-Gang Wen et al. Phys. Rev. C 78035805 (2008)

[31] Q. Wen et al. J.Phys. G: Nucl. Part. Phys., 38, 085103 (2011)

[32] M. La Cognata et al., Eur. Phys. J. A 27249 (2006)

[33] A. Rinollo et al. Nucl. Phys. A 758146 ( 2005)

[34] Li Chengbo et al. Phys. Rev. C 92025805 (2015)

[35] Li Chengbo et al. Phys. Rev. C 95035804 (2017)

[36] M. La Cognata et al., Phys. Rev. C 76065804 (2007)

[37] M. La Cognata et al., Phys. Rev. Lett. 101152501 (2008)

[38] M. La Cognata et al., Astrophysical J. 708796 (2010)

[39] A. Tumino et al. Phys. Lett. B 700111 (2011) 
[40] A. Tumino et al. Phys. Lett. B 705546 (2011)

[41] A. Tumino et al., Astrophysical J. 78596 (2014)

[42] M. La Cognata et al., Astrophysical J. Lett. 739 L54 (2011)

[43] I. Indelicato et al., Astrophysical J. 84519 (2017

[44] M.L. Sergi et al., Phys.Rev. C $R 82032801$ (2010)

[45] C. Spitaleri et al., Eur. Phys. Journ. A7 181 (2000)

[46] M. Gulino et al., J.Phys. G: Nucl. Part. Phys., 37125105 (2010)

[47] S. Cherubini et al., Phys.Rev. C R92 015805 (2015)

[48] R.G. Pizzone et al., Astrophysical J. 83657 (2017)

[49] L. Lamia et al., Astrophysical J. 850175 (2018)

[50] A. Tumino et al., Nature 2018 in press 